\title{
The Relation of Sex-associated Facial Profile Reversal and Stages of Human Palatal Closure '
}

\author{
ALPHONSE R. BURDI AND RALPH G. SILVEY \\ Department of Anatomy, University of Michigan, Ann Arbor, \\ Michigan 48104
}

\begin{abstract}
This report is concerned primarily with the chronological relations among stages of palatal closure, changes in facial profile, and the sex of a select group of human embryos representing the critical period of palate development. Histological serial sections of 40 human embryos ranging in age from 6 to 9 weeks and free from gross defects were studied using light microscopy and graphic reconstructions. Maxillary prognathism usually occurred concomitantly with vertically oriented palatal shelves. As the shelves progressively assumed a horizontal position prior to palatal closure, a profile reversal occurred, i.e., the lower jaw became prognathic. Both profile reversal and reorientation of the palatal shelves occurred earlier in males (seventh week) than females (eighth week). These patterns suggest a sexual dimorphism in which the palatal shelves in females are apart and vertical for a relatively longer time than in males. Also, the concordance between palatal-shelf elevation and mandibular prognathism suggests that the forward repositioning of the lower jaw may contribute to the removal of the tongue from between the palatal shelves.
\end{abstract}

The role of genetic factors has become an increasingly important consideration in the understanding of normal and abnormal facial development. It has been demonstrated that the frequency of human cleft palate is greater in females than males (Ivy, '57; Greene, '63; Milham, '63; Meskin et al., '65). Few attempts, if any, have been made to relate this sex difference with the possible occurrence of sex-associated prenatal variations. Recently Meskin et al. ('68) addressed themselves to this question and designed a hypothetical model that attempted to explain why females have a greater incidence and severity of palatal clefting. Their model assumed that the palatal shelves of human female embryos are apart for a longer time than those of males. This assumption was subsequently supported by a study (Burdi and Silvey, '69) that showed that females lag behind males in palatal closure. This finding implies (1) that sex-associated developmental patterning may operate in prenatal human facial development, and (2) that female embryos experience a longer period during which the normal sequence of palatal closure can be interrupted by teratogenic or other intrauterine disturbances.
These observations (Burdi and Silvey, '69) raise the question as to whether sexual variations can be observed in other facial regions before birth. For this purpose sexual variations in the embryonic human facial profile were sought during the period of palatal formation. Before the present study profile changes in the embryonic human face received only cursory attention, without specific reference either to sample size or sex. What is known about prenatal facial profiles stems primarily from Retzius ('04) who showed that before the seventh week the upper jaw is prognathic relative to the lower jaw but that this relation is temporarily reversed at about the ninth week. Later work (Schultz, '25; Schwarz, '31) supplemented the earlier study by showing that the anteroposterior profile of the lower jaw was generally recessive from the end of the second month until birth. Rabkin ('52) subsequently related variations in jaw profiles with ossification of the embryonic facial bones. These studies of prenatal

1 This study was supported in part by National Faculty Research Grant (34508), Project 21 from the Horace H, Rackham Graduate School, the University of Michigan, and Public Health Service Research grant of Michigan, and Public Health Service Research grant No. 5 (SO-1 FR5321) from the General Research Sup-
port Branch, Division of Research Facilities and Resources, to R.G.S. 
facial profiles were qualitative and did not consider sex differences. The significance of the association of inheritance, variable facial profiles, and critical periods of mammalian facial embryogenesis was recently emphasized by the demonstration of topographical and growth differences in early facial primordia (Trasler, '68) and timing differences in anteroposterior profile changes (Smiley, '68) in two different strains of mice.

The present study was designed to answer three questions. First, how early in human development can regular variations in facial development be recognized? Second, if such variations are observable, are they sex-associated? And, third, how might such prenatal variations be related to the mechanisms of both normal and abnormal human facial embryogenesis?

\section{MATERIALS AND METHODS}

Forty human embryos from the Embryology Collection of the University of Michigan Department of Anatomy were studied. Twenty were male and $20 \mathrm{fe}-$ male. The specimens ranged from 19 to $36 \mathrm{~mm}$ crown-rump length (CR) and from approximately 6 to 9 weeks of intrauterine age. This sample represented the secondary palate from the time the palatal shelves are first observable to later stages of palatal closure. Only embryos free of storage deformation and gross defects of the head and face were used.

The embryos were fixed in $10 \%$ neutral buffered formalin, paraffin embedded, and sagittally sectioned. Serially mounted sections were stained with either hematoxylin and eosin or trichromatic tissue stains, e.g., Mallory.

In order to minimize observer bias all embryos were first cephalometrically analyzed for upper and lower facial depths and then their sex was identified.

Facial depth and profile analyses. Light microscopy was used to identify the serial section that best passed through the combination of midline nasal septum, cranial base, and developing pituitary gland. Little if any distortion of the jaws was observed. The selected sagittal section was then projected, the image, magnified $25 \times$, was drawn, and measurements were made on the tracing of each projected image. Upper facial depth was measured by a line connecting the center of the pituitary gland mass with the midline incisor dental lamina of the upper jaw. Lower facial depth was measured by a line connecting the same pituitary landmark with midline dental lamina of the lower jaw. Data were recorded, in millimeters, as either upper or lower facial depths. The relation between the two measurements was shown by dividing each upper facial depth measurement by its lower jaw value.

Sex identification. According to Gillman ('48) and Burdi and Silvey ('69), sex of human embryos can be determined by observing the progressive specialization of the indifferent-stage gonad into either testis or ovary. The male gonad can be identified as early as $14-15 \mathrm{~mm} \mathrm{CR}$, i.e., at the time when the palatal shelves are first recognizable as projections from the lateral walls of the oronasal cavity. Differentiation of the testis is generally much more clear-cut than is that of the ovary. The key criteria used to identify the testis were (1) relative prominence of deep-staining seminiferous tubules or cords throughout the organ, which were separated by spindleshaped mesenchymal cells, (2) decrease in the number of primordial cells in the cellular zone of the testis, and (3) thickened outer covering (tunica albuginea) of the organ. Presence of the seminiferous tubule system was the most important and clearcut criterion used. Identification of the ovary was made chiefly by negative morphological features. That is, absence of a testis and presence of a young ovary were ascertained by noting a relative lack of seminiferous tubules and by a relatively thin outer covering of the organ. The most important criterion for identifying ovaries was the persistence of a large number of primitive sex cells in the outer cellular zone of the differentiating gonad.

\section{FINDINGS}

Data pooled from all embryos indicated a synchrony between facial profile and palatal-shelf orientation, with vertical positioning of the palatal shelves being related to a prognathic upper facial profile. With progressive movement of shelves from a 
vertical to horizontal position a reversal was noted, i.e., the lower jaw profile became prognathic relative to a recessive upper facial shape. This pattern was apparent from the time the palatal shelves were first recognizable, at approximately 6 weeks, until the palate completely closed.

Differences in this general pattern were noted when the sample was divided according to sex. Male embryos of 6 to 7.5 weeks (19-25 mm CR) possessed palatal shelves that were positioned vertically on either side of the tongue (fig. 1, table 1). This age group, except for two cases, showed a maxillary depth greater than the depth of the lower jaw. The maxillary prognathism was best demonstrated by noting that the ratio, on a percentage basis, of upper facial depth to lower facial depth was more than 100 . In older specimens up through the age of the oldest male specimen in the series there was a progressive anterior to posterior gradient in which the originally vertically oriented palatal shelves moved into a horizontal position. As noted in figure 1 this shelf repositioning occurred concomitantly with a facial profile reversal in which the lower jaw became prognathic and the upper jaw recessive.

Similar developmental patterns regarding timing of shelf repositioning and profile reversals were observed in the female embryos. But relative to the males the females showed a noticeable time lag in this change (fig. 2, table 2), since they showed a vertical palatal shelf orientation and a prognathic upper facial profile until the eighth week. It was not until this time that the female palatal shelves became progressively horizontal and facial profiles shifted from a prognathic upper face to a prognathic lower face.

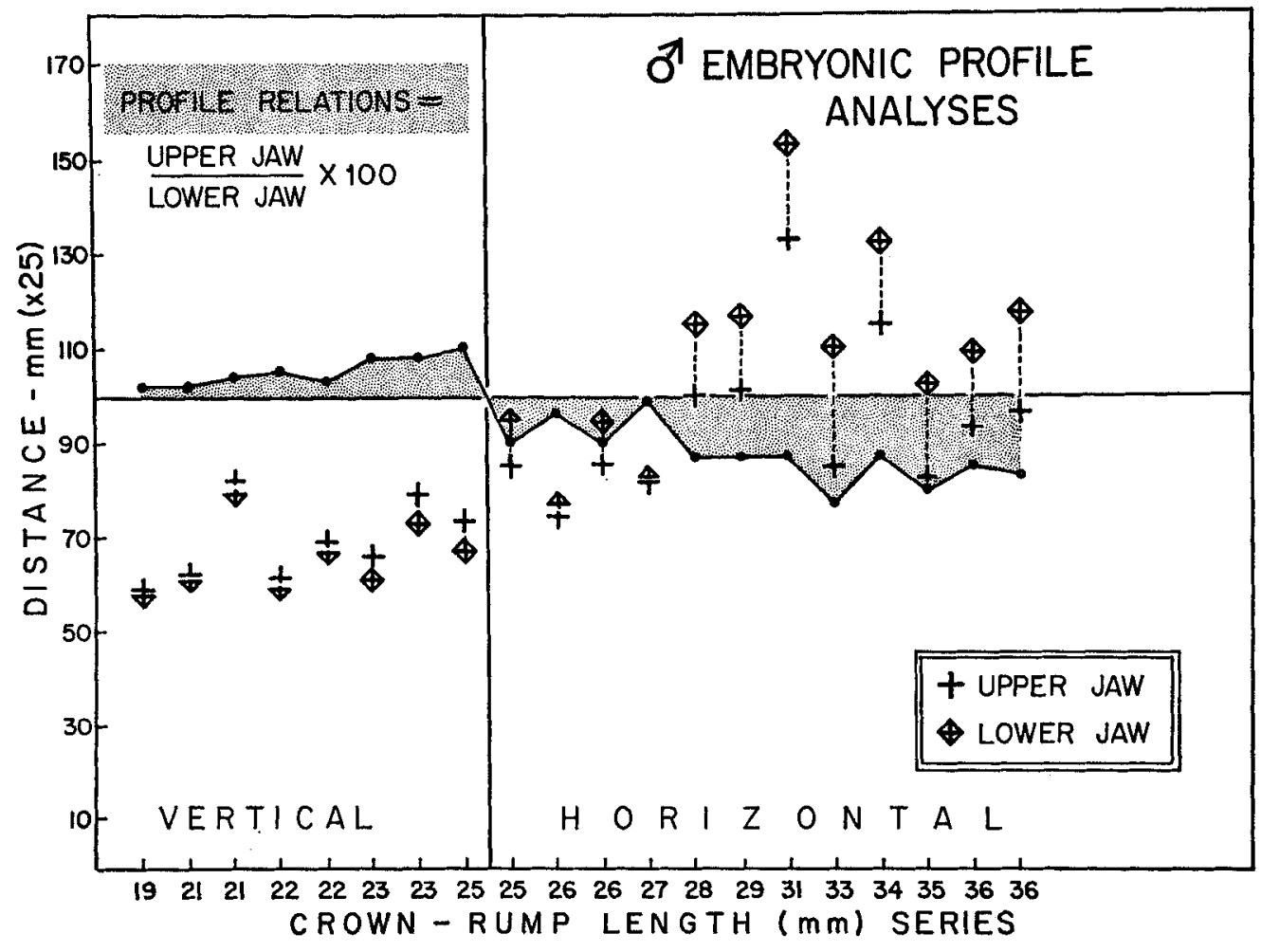

Fig. 1 Male embryonic profile analyses. In addition to showing the relation between profile change and crown-rump length, changing upper and lower jaw relations are shown as the stippled area around the $100 \%$ line. 
TABLE 1

Male embryonic facial profile analyses ${ }^{\text {: }}$

\begin{tabular}{|c|c|c|c|c|c|}
\hline \multirow{2}{*}{ Specimen } & \multirow{2}{*}{ Crown-rump } & \multicolumn{2}{|c|}{ Facial depths } & \multirow{2}{*}{$\begin{array}{l}\text { Profile } \\
\text { relation }= \\
\begin{array}{c}\max \div \operatorname{mand} \\
\times 100\end{array}\end{array}$} & \multirow{2}{*}{$\begin{array}{c}\text { Shelf } \\
\text { position } 2\end{array}$} \\
\hline & & Maxillary & Mandibular & & \\
\hline & $m m$ & $m m$ & $m m$ & & \\
\hline 1 & 19 & 31 & 58 & 102 & V \\
\hline 2 & 21 & 62 & 61 & 101 & V \\
\hline 3 & 21 & 82 & 79 & 104 & $\mathbf{V}$ \\
\hline 4 & 22 & 62 & 59 & 105 & V \\
\hline 5 & 22 & 69 & 67 & 103 & V \\
\hline 6 & 23 & 66 & 61 & 108 & V \\
\hline 7 & 23 & 79 & 73 & 108 & $\mathrm{~V}$ \\
\hline 8 & 25 & 74 & 67 & 110 & V \\
\hline 9 & 25 & 85 & 94 & 90 & $\mathrm{H}$ \\
\hline 10 & 26 & 74 & 77 & 96 & $\mathrm{H}$ \\
\hline 11 & 26 & 85 & 94 & 90 & $\mathrm{H}$ \\
\hline 12 & 27 & 81 & 82 & 99 & H \\
\hline 13 & 28 & 100 & 115 & 87 & $\mathrm{H}$ \\
\hline 14 & 29 & 101 & 116 & 87 & $\mathrm{H}$ \\
\hline 15 & 31 & 133 & 153 & 87 & $\mathbf{H}$ \\
\hline 16 & 33 & 85 & 110 & 77 & $\mathbf{H}$ \\
\hline 17 & 34 & 115 & 132 & 87 & $\mathrm{H}$ \\
\hline 18 & 35 & 85 & 102 & 83 & $\mathbf{H}$ \\
\hline 19 & 36 & 93 & 109 & 85 & $\mathrm{H}$ \\
\hline 20 & 36 & 97 & 117 & 83 & $\mathbf{H}$ \\
\hline
\end{tabular}

1 Measurements, except crown-rump length, are $25 \times$ actual.

$2 \mathrm{~V}$ and $\mathrm{H}$ indicate vertical and horizontal palatal-shelf orientation, respectively,

\section{DISCUSSION}

This study showed that in young human embryos there is a relation between facial profile reversal and the key stages of palatal closure, and, moreover, that this relation appears to be a function of time and sex. These findings are especially significant in terms of the etiology or production of such common facial defects as cleft palate.

First, it is important to recall the probable timing of human palatal closure. Fulton ('57) showed that the critical period of human palatal-shelf closure is about 8 weeks ( $30 \mathrm{~mm} \mathrm{CR}$, range $29-33 \mathrm{~mm}$ ). No reference was made to sex of the embryos. This study was significant because it provided precise information of the timing of events involved in palatal closure.

Recent epidemiological studies of the sex differences in frequency of cleft palate have drawn attention to still another need for developmental data that goes beyond the simple consideration of timing and palatal-closure sequences. The sex-associated parameter of human palatal development demonstrated by us is relevant to explanations of sex differences in fre- quency of isolated cleft palate. The pooled 619 clinical cases reported by Knox and Braithwaite ('63), Fogh-Anderson ('43), and Mazaheri ('58) showed a 1.5:1 female to male ratio for palatal clefts of all types, a 2:1 female to male ratio for isolated clefts of the secondary palate, and a 1.2:1 ratio for clefts of the soft palate. The question arose from these studies as to why females show a greater incidence and severity of palatal clefting.

Meskin et al. ('68) recently recognized the possible occurrence of sex-associated differences in human facial development in attempting to explain the sexual dimorphisms observed in clinical cases of palatal clefts. They proposed a hypothetical model explaining such differences on the basis of the onset of palatal closure in female human embryos lagging behind that in males. This hypothesis suggested that in females the palatal shelves are apart for a longer period of time than in males. Females would therefore experience a greater period of teratogenic susceptibility or period of potential disruption of normal prenatal development. 


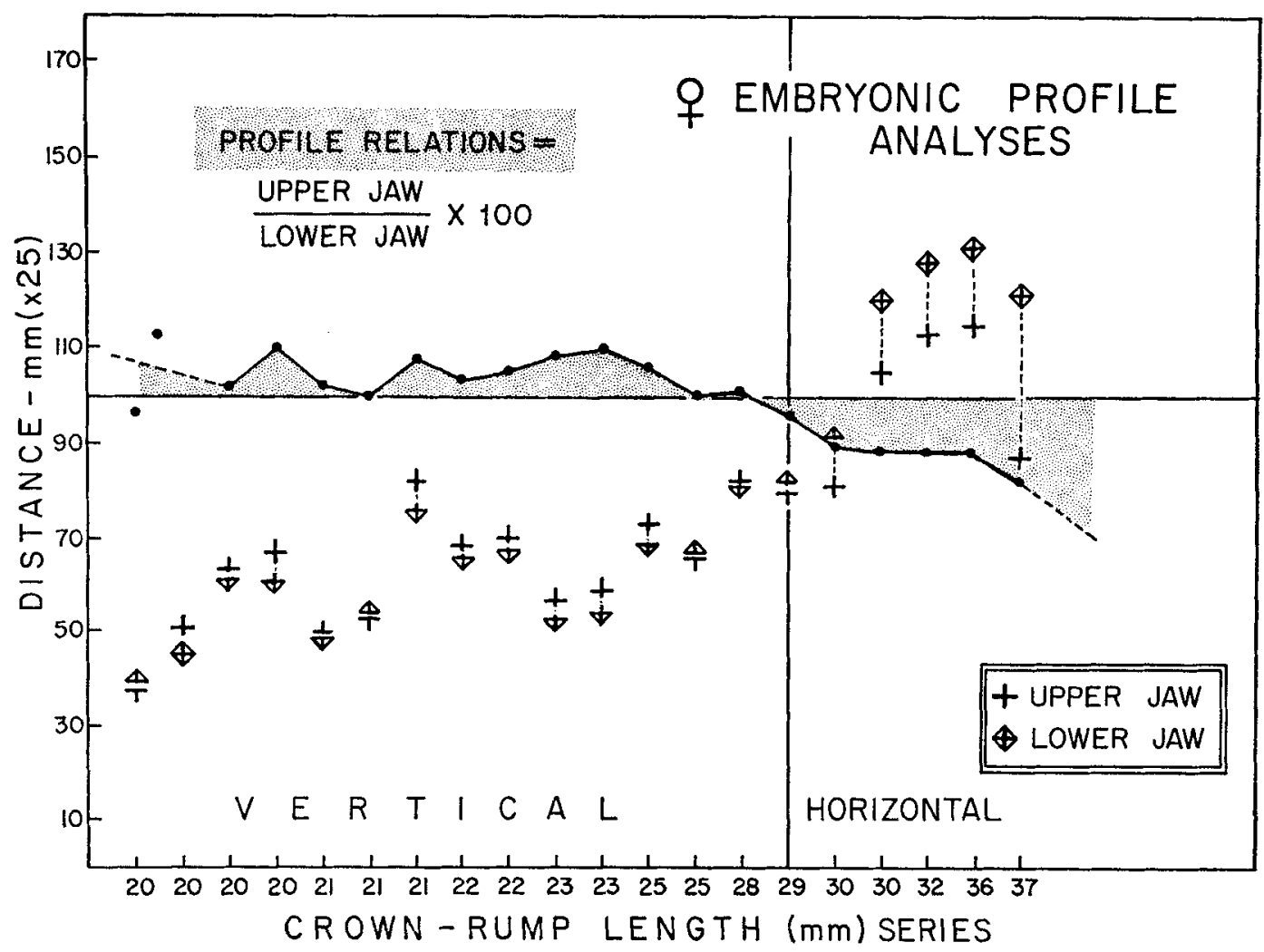

Fig. 2 Female embryonic profile analyses. In addition to showing the relation between profile change and crown-rump length, changing upper and lower jaw relations are shown as the stippled area around the $100 \%$ line.

As a follow-up to this model, Burdi and Silvey ('69) related time-linked stages of palatal closure with embryonic sex. In 46 embryos they reported that palatal shelves of males begin closing earlier ( 25 $\mathrm{mm} \mathrm{CR}$ or early seventh week) than do those of females, which closed beginning at the mid-eighth week. The importance of short-interval timing and gene-determined differences in mammalian development was well demonstrated in various strains of mice (Fraser, '55; Walker and Fraser, '56). Our earlier study thus provided data supporting the suggestion that sexual dimorphisms may normally occur in human facial development.

The question now arises as to the significance of facial profile changes in normal and abnormal human palatal development. Although a study of human embryos is limited chiefly to determining develop- mental associations rather than causal relations, the temporal associations of palatal closure stages and profile changes prompt some discussion of mechanisms.

The present study showed that (1) prior to movement of the palatal shelves into the horizontal position the upper face is prognathous relative to the lower jaw; (2) this maxillomandibular-profile relation is reversed with the onset of horizontal shelf positioning; (3) following this early period of normal palatal closure the lower jaw once again becomes recessive in profile; and (4) this pattern of shelf movement and profile reversal occurs earlier in human male than female embryos. The last observation complements earlier subjective reports by Retzius ('04), Schultz ('25), Schwarz ('31), and Rabkin ('52).

Various mechanisms, at the macroscopic level, have been postulated for palatal clos- 
TABLE 2

Female embryonic facial profile analyses ${ }^{1}$

\begin{tabular}{|c|c|c|c|c|c|}
\hline \multirow{2}{*}{ Specimen } & \multirow{2}{*}{ Crown-rump } & \multicolumn{2}{|c|}{ Facial depths } & \multirow{2}{*}{$\begin{array}{l}\text { Profile } \\
\text { relation }= \\
\begin{array}{c}\max \div \text { mand } \\
\times 100\end{array}\end{array}$} & \multirow{2}{*}{$\begin{array}{c}\text { Shelf } \\
\text { position } 2\end{array}$} \\
\hline & & Maxillary & Mandibular & & \\
\hline & $\boldsymbol{m m}$ & $m m$ & $m m$ & & \\
\hline 1 & 20 & 38 & 39 & 97 & $\mathbf{V}$ \\
\hline 2 & 20 & 51 & 45 & 113 & $V$ \\
\hline 3 & 20 & 63 & 62 & 101 & $\mathbf{V}$ \\
\hline 4 & 20 & 67 & 61 & 109 & V \\
\hline 5 & 21 & 50 & 49 & 102 & V \\
\hline 6 & 21 & 53 & 53 & 100 & $\mathbf{V}$ \\
\hline 7 & 21 & 82 & 76 & 107 & $\mathrm{~V}$ \\
\hline 8 & 22 & 68 & 66 & 103 & V \\
\hline 9 & 22 & 70 & 67 & 104 & $\mathbf{V}$ \\
\hline 10 & 23 & 57 & 53 & 107 & $\mathbf{V}$ \\
\hline 11 & 23 & 59 & 54 & 109 & V \\
\hline 12 & 25 & 66 & 66 & 100 & $\mathbf{V}$ \\
\hline 13 & 25 & 73 & 69 & 106 & $\mathbf{V}$ \\
\hline 14 & 28 & 82 & 81 & 101 & $\mathbf{V}$ \\
\hline 15 & 29 & 79 & 82 & 96 & $\mathbf{V}$ \\
\hline 16 & 30 & 81 & 91 & 89 & $\mathbf{H}$ \\
\hline 17 & 30 & 105 & 120 & 87 & $\mathbf{H}$ \\
\hline 18 & 32 & 113 & 128 & 88 & $\mathbf{H}$ \\
\hline 19 & 36 & 115 & 131 & 88 & $\mathrm{H}$ \\
\hline 20 & 37 & 87 & 121 & 72 & $\mathbf{H}$ \\
\hline
\end{tabular}

1 Measurements, except crown-rump length, are $25 \times$ actual.

$2 \mathrm{~V}$ and $\mathrm{H}$ indicate vertical and horizontal palatal-shelf orientation, respectively.

ure, among which are intrinsic shelf forces (Walker and Fraser, '56; Fraser, '67), contraction of the tongue downward from between the palatal shelves, and onset of orofacial muscular activity (Humphrey, '68). Although combinations of these suggested mechanisms may operate at specific times the observation of a relative "overgrowth" in mandibular length may be another mechanism by which the tongue is removed from between the vertically oriented palatal shelves allowing them to reposition themselves in the horizontal position before palatal closure. As a purely speculative effort, failure of this mandibular prognathism to occur during the stages of palatal closure may be one explanation for the clefting seen in clinical defects involving both mandibular micrognathism and palatal clefting, i.e., Pierre Robin syndrome. Another interesting observation involves the coordination between palatalshelf positioning, profile reversal, and progressive specialization of the nasal capsule supporting the soft-tissue matrix of the upper face and palatal region. Prior to horizontal positioning of the palatal shelves the nasal capsule exists primarily as a dense-staining prechondral mass of mesenchyme. As the shelves are repositioned and the lower jaw assumes a prognathic profile the nasal capsule becomes cartilaginous, as evidenced by the presence of typical cell lineage of chondrocytes. Whether this soft tissue-capsular relation is more than a coincidence in unknown, but it has been shown that defective growth in length of the cartilaginous nasal capsule can be closely associated with clefts of the secondary palate (Avery, '61).

\section{LITERATURE CITED}

Avery, J. K. 1961 The nasal capsule in cleft palate. Anat. Anz., 109: 722-726.

Burdi, A. R., and R. G. Silvey 1969 Sexual differences in closure of the human palatal shelves. Cleft Pal. J., 6: 1-7.

Fogh-Anderson, P. 1943 Inheritance of Harelip and Cleft Palate. Munksgaard, Copenhagen.

Fraser, F. C. 1955 Thoughts on the etiology of clefts of the palate and lip. Acta Genet., 5: 358-369.

1967 Cleft lip and cleft palate. Science, 158: 1603-1606.

Fulton, J. T. 1957 Closure of the human palate in embryo. Am. J. Obst. Gyn., 74: 179-182.

Gillman, J. 1948 The development of the gonads in man, with a consideration of the 
role of fetal endocrines and histogenesis of ovarian tumors. Carnegie Contr. Embryol., 32: 81-131.

Greene, J. 1963 Epidemiology of congenital clefts of the lip and palate. Publ. Hlth. Rep., $78: 589-602$.

Humphrey, T. 1968 The development of mouth opening reflexes involving the oral area of human fetuses. Alabama J. Med. Sci., 5: 126157.

Ivy, R. H. 1957 Congenital anomalies as recorded on birth certificates. Plast. Reconstr. Surg., 20: 400-411.

Knox, G., and F. Braithwaite 1963 Cleft lips and palates in Northumberland and Durham. Arch. Dis. Child., 38: 66-70.

Mazaheri, M. 1958 Statistical analysis of patients with congenital cleft lip and/or palate at the Lancaster Cleft Palate Clinic. Plast. Reconstr. Surg., 21: 193-203.

Meskin, I. H., R. J. Gorlin and R. J. Isaacson 1965 Abnormal morphology of the soft palate. II. The genetics of cleft uvula. Cleft Pal. J., 2: 40-45.

Meskin, L. H., S. Pruzansky and W. H. Gullen 1968 An epidemiologic investigation of factors related to the extent of facial clefts. I. Sex of the patient. Cleft Pal. J., 5: 23-29.
Milham, S. 1963 Underreporting of incidence of cleft lip and palate. Am. J. Dis. Child., 106: 185-188.

Rabkin, S. 1952 Variations in structural morphogenesis of the human face and jaws. J. Dent. Res., 31: 535-547.

Retzius, G. 1904 Zur Kenntnis der Entwicklung der Körperform des Menschen während der fetalen Lebensstufen. Biol. Untersuch., Neue Folge, 11: 32-76.

Schultz, A. D. 1925 Studies on the evolution of human teeth. Dent. Cosmos, 67: 935-947.

Schwarz, A. M. 1931 Untersuchungen über die embryonale Progenie. Fort. Orthodont., 1: 821.

Smiley, G. R. 1968 A comparison of normal growth parameters of the craniofacial complex in C57BL and $\mathrm{A} / \mathrm{Jax}$ mice during palatal closure. Abst., 26th ann. mtg., Am. Cleft Pal. Ass.

Trasler, D. G. 1968 Pathogenesis of cleft lip and its relation to embryonic face shape in $A / J$ and C57BL mice. Teratology, 1: 33-49.

Walker, B. E., and F. C. Fraser 1956 Closure of the secondary palate in three strains of mice. J. Embryol. Exp. Morph., 4: 176-189. 